\title{
PORTRAYING THE TURN SIGNAL MARKERS USE AMONG NON-NATIVE AND NATIVE SPEAKERS OF ENGLISH (A PRAGMATIC PERSPECTIVE)
}

\author{
Udi Samanhudi \\ Tirtayasa State University (UNTIRTA)
}

\begin{abstract}
It has been known that among many activities, conversation is one activity which is very potential to build up social relationships and exchange ideas among many people in their community. This fact indicates that conversation plays a crucial role in everybody's life. For a good conversation to take place, the awareness of the speakers to sign that they are finishing and that the listeners begin a response seems to be one of the key points to succeed the conversation activity. This means that the participants, both speakers and hearers, should apply turn signals as indication of willingness to take, hold, or pass the floor during the course of the conversation. This writing is a report of a minor study on turn signals in English conversations done by Indonesians as non-native speakers and Americans as native speakers of English who are found to use English in their conversations. The English conversations analyzed in this study are those conversations taking place in natural contexts like in a lobby of a hotel, a workshop room and a restaurant. Those conversations are recorded, transcribed and analyzed. In order to get the whole picture of both the turn signal markers used and their purposes, a participatory observation was also conducted. Thus, to be more focused, this research is limited to the analysis of markers used by both Indonesians and Americans in tum-taking. The results reveal that both Indonesians and Americans use almost the same markers (words) in tumtaking like yeah, well, you know, I think, well yeah, etc. Those markers are applied interchangeably in the turn-taking done by the two groups in the course of the conversations done. In terms of purpose, the use of those markers in turn-taking is mainly to indicate that one of the participants in the conversations (both NNS and NS) wants to take the floor and have the talking turn. The difference found between the two groups was pause (silent) in signaling something in a conversation being done especially when one of
\end{abstract}


them is talking. Indonesian tends to make a longer pause to show, probably, that they are attentive to their colleague having the turn of speaking. This might be done because keep silent when other people are talking is considered polite. Meanwhile, the two Americans involved in this study keep using certain words of signaling such as well, yeah, hmm, during the course of the conversations including when one of them is talking. This seems to be done in order to avoid the sense of being rude or impoliteignoring the one having the turn of talking. Finally, this could be done also to indicate that they are paying attention to what their colleague/s say and keep the conversation runs smoothly. From this, it is clear that, cultural normswhat they believe about being polite- also play a role in turn taking activities in a conversation.

Keywords: Turn signals, English conversation, pragmatic perspective

\section{A. Background of the Study}

Pragmatic concern on cooperative communication has once been proven by the idea of Cooperative Principle (CP) proposed by Grice as cited in Thomas (1995). According to him, in order to have an effective communication, which in this study, refers to the success of making use of the turn signal markers to gain, hold or yield the floor (Rahman, 2006), participants should fulfill the four maxims proposed- quality, quantity, relation and manner. Following these four maxims, the speakers will need to show the genuineness of providing the information, provide the required amount of information, the relevance and the way of telling the information (Huang, 2007, Yule 1994 and Thomas, 1996).

With regard to the turn signals in English conversations, many studies indicate the importance of turn signal and understanding it in order to succeed a conversation. A study conducted by Rahman (2006), for example, revealed that the success of turn signals used by!ndonesian speakers in speaking English determine the success of the conversation being done. To his finding, most Indonesians used the four types of turn signals - turn-claiming, turn-yielding, turn-taking and turnholding in their conversations. Thus, according to him, the use of those turn signals appropriately would determine the smooth exchange of the English conversations being done. In addition, Murdoch (2000), conducted a study on turn-taking strategies in Korean English conversation. In her study, it is found that many Koreans failed to use the turn signal such as turn -taking strategies in English due to 
the absence of certain turn-taking strategies of English in Korean language. She further said that more real examples of tum signals of English are needed so that Koreans could successfully interact in English especially in their interaction with people coming from all over the globe. Thus, the two studies show that turn signal in an English conversation is important to be further investigated in order to give a deeper insight on the turn signals types and markers commonly used in a conversation both by native speakers of English (NS) as well as non- native speakers of English (NNS) (Orestrom as cited in Rahman, 2006).

Departing from those facts illustrated above, this research is aimed at describing the turn signal markers used by both non-native speakers (Indonesians) and native speakers of English (Americans) as well as their purposes of using those markers. For this study to take place, two questions are proposed. First, what markers are used by both non-native and native-speakers of English in signaling a turn? Second, do they use those markers for the same purposes? by answering these two questions, this study could help learners of English to fully understand the turn signal markers which have been a troublesome for learners of English as a foreign language, owing to its socio cultural component and various turn-eliciting cues (Coulthard, 1977). However, the finding of this research might not be able to represent the whole picture of turn signal markers due to the limited number of participants involved in this study and limited access to a more various natural contexts of situation of English conversations used in this research. The researcher got a limited access to those various contexts of conversational settings due to the time and location constraints.

\section{B. Theories Informed the Study}

\section{Turn Signals in Conversation}

Turn signal in conversation is commonly used to keep the conversation going. By signaling the turn, each participant can take a role both as a speaker and as a hearer. By this, a "fair" communication could take place. In response to this, Orestrom (1983) says that a successful exchange in a conversation is determined by the awareness of the speaker to give a sign that he/she is finishing and that the listener begins a response. This shows us that turn signal plays a crucial role in a conversation being done. In line with this, McDonough and Shaw say the importance of turn signals as follows. 
"Within the framework of the conversation, "tums" have to take place if the conversation is not to be totally one-sided. Certain strategies like turntaking, turn-holding have to be put into operation by the speaker" (McDonough and Shaw, 1993: 156)

What McDonough and Shaw say above indicates that a "fair" play in a conversation is important so that it is not creating a one-sided communication which is surely "unfair" for one of the participants of the conversation itself. In other words, each participant has to have an equal role in the conversation and therefore, knowing when and how to take a turn and hold the floor is absolutely vital. Implied in this last statement is the importance of knowing the turn markers for non native speakers especially those turn signal markers made by native speakers of English for a more effective, say, intercultural communication. As Tibbetts (2007) says, turn signal markers use is really cultural.

With regard to the crucial role of turn signal in a conversation, Cook (1989) emphasizes the importance of applying the turn signals appropriately and effectively in order to avoid violating the turn exchange conventions, the violation of which may result in unsmooth flow of exchanges (p. 52). This means that only knowing the turn signals is not enough, knowing how to apply them is also important. In other words, being familiar with markers to be used in signaling a turn and being familiar with the appropriate use of those markers are very important.

According to Poyatos as cited in Rahman (2006), there are five types of turn signals: turn-claiming, turn-yielding, turn-taking, turn-holding and turnsurpressing. Turn claiming signal is a signal made by a listener to indicate that he or she wants to talk, for instance but ..., I ...., or one minute ... Turn yielding, on the other hand, occurs when the speaker feels he or she has gone on long enough, when his/her listeners have claimed or briefly taken the floor. It is signaled by phrases such as yes, go ahead. Turn-taking signal occurs when a listener takes the floor after the speaker shows his or ber willingness to relinguish his turn, or when his or her turn-claiming has failed and he or she just decides to take it. The expressions like I was going to say that ..., yeah, well, uh, and no, are some examples of tumtaking signals. Turn-holding signal is a signal which attempts to surpress a turn claim with, for example, something like let me finish ... and wait. Turn-surpressing signal is related to turn-holding but it can be started by any of those involved in conversations and not simply by the one who is currently occupying the floor, for example, let him finish, and listen (p. 10). 
In terms of culture and social values, Cook (1982) argues that the differences in the kinds of signals and the markers used that occur during a conversation are not only fostered by the difference of cultural values and social meaning between one particular language with others, but may also be caused by individual differences among the speakers. Thus the turn signal markers used are characteristically multifunctional with a variety of pragmatic or discourse functions which depend on the context. Therefore the search for a core meaning of pragmatic markers constraining their multifunctionality is an important issue in research on the semantics/pragmatics interface (Ajjmer, 2002).

In addition, According to Duncan, cited in Coulthard (1977: 68), a listener in a conversation may claim the speaking turn when the current speaker (the one holding the floor) gives a turn signal. Thus, the indications or cues of the turn signals could be seen below:

1. Intonation : the use of any pitch level/terminal juncture combination, other than 22 .

2. Paralanguage: drawl on the final syllable or on the stressed syllable of the phonemic clause.

3. Body motion: the termination of any hand gesticulation or the relaxation of a tensed hand position.

4. Sociocentric : the appearance of one of several stereotyped expressions, such as 'but, uh', 'or something', 'you know', labeled as socio centric .

5. Paralanguage: a drop in paralinguistic pitch and/or loudness, in conjunction with socio centric sequence.

6. Syntax : the completion of a grammatical clause involving a subjectpredicate combination."

In this study, the focus of turn signals cues used is the cues belong to the socio centric sequences such as "but, $u h$, you know, and so on. Those signals are categorized into four turn signals which are turn-claiming, turn-yielding, turntaking and turn-holding. However, to be more focused, this study only analyzes the turn-taking markers and purpose of using those markers by both non-native speakers and native-speakers of English involved in the conversations used in this study. Finally, the context of situation, linguistics and physical are used in order to identify possible reasons for why participants involved in the conversation using such turn signal markers. 


\section{Context and Conversational Analysis}

Pragmatic concern on the importance of context in studying a language in use has been proven by Yule (1996) through his definition of pragmatic. In his very simple but concise definition, Yule said that pragmatic is the study of language in use. This implies that context surrounding the use of the language in form of, for example, conversation, is important in order to fully understand what is intended by a speaker to his/her hearer in a conversation .

In addition, the importance of making use of context in a conversational analysis has long been believed by many other experts. Eggin (2004), for example, said that in order to make a sense of a text (could be a conversational text), it may not only come just from within the textual environment but also from the extratextual context. The context here could refer to the context of situation and culture. In addition, Sternstorm (1994) said that the utterances of a speaker in a conversation are not isolated phenomena but depend on the entire context for their interpretation. She further added that in interpreting what is exactly meant by a speaker, not only the immediate context referring to what the previous speaker just uttered but also the wider context which includes the speech situation, the topics, the speakers and the relationship to each other. From this explanation, it could be noticed that context plays a crucial role in understanding a language use phenomenon including conversation.

Following Anonym (2008) available on line at http://www.unc.edu/ gerfen/Ling30Sp2002/pragmatics.htm, context is divided into four subareas physical context, epistemic context, linguistic context and social context. Physical context refers to where the conversation is taking place, what objects are present, what actions are occurring, and so on. Epistemic context is about what speakers know about the world. For example, what background knowledge is shared by the speakers is crucially part of our epistemic knowledge when we have a conversation with someone else, etc. Linguistic context, in addition, is related to what has been said already in the utterance. Finally, social context refers to the social relationship among speakers and hearers.

In this study, three context subareas are used in order to interpret the meaning and (possible) reasons for certain use of turn signals by participants of conversation analyzed in this study. The researcher describes the place where the conversation takes place, the choice of words during the conversation by each participant and the social relationship among conversation participants involved. 


\section{Research Method}

This study is a descriptive study as it is aimed at portraying the use of turn signal markers among non-native (two Indonesians) and native speakers of English (two Americans) who are involved in this study. It is focused on describing the similarities of turn signal markers used during the conversation done by the two groups. To be more focused and detailed, the turn signal markers analyzed are limited to the turn taking in informal conversation done in natural contexts of situations such as in a lobby of a hotel (during breakfast time), workshop room and restaurant. The subjects of the study are two Indonesians who were found to talk in English and two Americans who were talking with their colleagues from both Indonesia and their own country (America).

In collecting the data, several techniques were used like recording, transcribing and categorizing the marker used to indicate a turn taking. The researcher first recorded the conversation directly and then transcribed each conversation. Categorization was made by confirming each marker of turn signal found to the characteristics of turn-taking based on Poyatos as cited in Loveday (1982). In order to get a full picture of the use of those turn signal (turn-taking) markers (well, yeah, so, etc), the researcher had a participatory observation during the progress of the conversations made by both Indonesians and Americans.

In analyzing the data, the researcher steadily moves among four stages: coding data, shuttling among reduction, displaying and drawing conclusion. The coding of data leads to new ideas what should go into the table. Entering the data requires further data reduction. As the table fills up, preliminary conclusions are drawn, but they lead to the decision to add another column to the table to test the conclusion. Thus, the data analysis is therefore a repetitious and iterative enterprise (Miles and Huberman, 1984). In brief, the data analysis employed in this research is as follows: The first step was to recognize and identified the verbal turn signals. Another step was to conduct a peer discussion and verification in order to have deeper insights into this study. This, in turn, became useful inputs for drawing conclusion pertaining to the provision of the verbal signals and the pragmatic functions they employed. 


\section{Finding and Discussion}

\section{Finding}

In general, the results of the analysis reveal that both Indonesians as non native and Americans as native speakers of English use almost the same turn signal markers in turn-taking such as well, ya, so, okay, sorry, I see, and yeah. These markers, in general, seem to be used to show that one of them wants to take the floor and at the same time keeps the smooth exchange of the conversation being done by those participants of the two groups involved in this study. However, the use of those markers, in terms of frequency, seems to be relatively different between the two. The Indonesians used the turn- taking markers such as well, right and sure, mostly in only turn-taking time. Meanwhile, the native speakers (Americans) tend to keep using those markers during the progress of the conversation and not always intended to take the turn of talking. The first could be caused by their belief on being "cooperative" as a listener. Meanwhile, for the latter, it is likely aimed to control, keep and appreciate the one having the floor due to their belief that keep signaling verbally indicated by the use of turn signal markers when one of them having a turn to talk. Below are tables recapping the words used to sign a turn and the occurrence of the words by both non-native and native-speakers of English.

Table I: Turn -Taking Markers used by Indonesians (NNS)

\begin{tabular}{|c|c|}
\hline $\begin{array}{c}\text { Turn-taking Markers among } \\
\text { Non-native Speakers }\end{array}$ & Number of Markers Used \\
\hline Well & 5 \\
\hline Yes & 6 \\
\hline Yes sure & 2 \\
\hline Well yeah … & 5 \\
\hline Well right . & 2 \\
\hline I see & 1 \\
\hline But & $\frac{4}{3}$ \\
\hline But yes & 1 \\
\hline So & - \\
\hline
\end{tabular}


F: <You know $>$, last night I checked the email about the hotel reservation in Bali and you know $<\ldots$ err .... > I had somebody to help me from the hotel but $<\ldots$.hm, well, $>$ I forgot to save the last email as I deleted some.

$\mathrm{K}:<$ Well, right, $>\mathrm{I}$ think I can ask somebody to help for this reservation. $<\mathrm{Mmm}$ ( to avoid pause) well, $>$ I have the number of the hotel $<$ and $\mathrm{hmm}>$ probably call it to night. (At the same time when $\mathrm{K}$ speaking, $\mathrm{F}$ keep saying yeah, hmm, right--)

F: <Well, okay> that's a good idea (laugh) so I do need to worry then $\mathrm{K}:<$ Yeah, well, > could be.

The conversation above shows that turn-taking is commonly signed by the use of certain markers. The use of yeah, well, hmm and right said by $\mathbf{K}$ indicates that he keeps the conversation going by showing his full attention to what $F$ said. This also means that the function of turn signals which is to maintain a conversation is relatively right. Other interesting fact is the signal words or markers which are found in almost every sequence of the conversation-well, right, well, okay, yeah and well yeah which are not always used to indicate a turn. This proves to us that one critical effort to maintain the conversation is keep signaling by using those certain/various markers during the progress of the conversation. In other words, the "signaling" efforts made by the two speakers above ( $F$ and $K$ ) could be a good way to keep the conversation run smoothly. The other example could be seen in the following conversation between $F$ and $K$ in a different context of situation.

Situation: This conversation was done by the two Americans ( $F$ and $K$ ) in a lobby of a hotel. They were discussing a possibility of providing a suggestion box for participant in the workshop room to be conducted in the following day.

F: <See>, I think it's important to have $<$ hmmm $>$ a kind of suggestion box for tomorrow.

$\mathrm{K}:<$ Right, right $>$

$\mathrm{F}:<$ You know $>$ the suggestion box will give participants more opportunities to, <well, you know, hmm> give comments, suggestions or things like $<\mathrm{hmm}>$ suggestion toward the issue and all things we give them tomorrow. So, $<\mathrm{hmmm}>$ I will ask committee from UM to provide it.

$\mathrm{K}:<$ Yeah, right, but I think, > <yeah> it doesn't mean that we do not ask them directly the comments or hmm question during the workshop yeah, I think it's it can give them direct response as well. 
In this conversation, each of the speakers uses the words see, right, you know, yeah, but I think, as their way of showing a turn. Thus, the same thing happens as that in the first conversation. Each of them keeps giving a signal of "paying attention" the one having the floor. This indicates that cultural belief in terms of "politeness" is relatively right. They seem to believe that signaling is an important part during the progress of the conversation made. This phenomenon is in line with what Brown and Levinson (1987) who said that in native speaker conversation signaling markers including those words used to sign a turn like well, yeah, I think, yes, well, etc have interpersonal function and are associated with face-saving, politeness and indirectness rather than with-imprecision, approximation or uncertainty.

However, although the words or markers used to sign a turn are relatively the same-yeah, well and so on, conversation done by the Indonesians involved in this study show a slight difference in terms of it way of turn-taking. Below is an extract taken from two Indonesians speaking in English.

Situation: Two Indonesians participating in the workshop were talking about the topic of the workshop about Action Research in Education. Each of them was asking about their understanding toward the topic which, according to them, was relatively new as they are so far concern only with Classroom Action Research.

$\mathrm{T}:<$ Well $>$, I see the workshop is interesting about Action research, do you enjoy that?

$\mathrm{H}:<\mathrm{Yes}>$, it is hmm (pause) it is interesting because we get lot of information about action research in education. So, (pause) it gives me, you know, let's say a new way of seeing hmmm ...the concept of Action Research. So, yeah, I think it's good and yeah very good.

(Tjust keeps silent when $H$ is talking)

$\mathrm{T}$ : I think I learn much about this concept. Well, you know, I think the concept is broader yeah I mean broader than the classroom action research that we, you know, so far know.

$\mathrm{H}:<$ Yes, yes sure>.

The conversation above was taking place in a workshop room in a hotel. The two participants shared about what they have got from the workshop. In terms of turn-claiming, the speaker $\mathrm{T}$ uses the word well to start the dialogue. This is 
interesting because the speaker ( $\mathrm{T}$ ) might assume that the hearer has got some ideas about what is going to talk about due to the shared context each of them has - stay at the same room and attend for the workshop. In other words, the speaker (T) sees the context of situation as an important cue for the hearer $(\mathrm{H})$ to understand the topic to talk or discuss. Further, another interesting fact is that not too many words are used to signal the turn. In the conversation above, $T$ just say "I think" as a response of what $H$ said previously about his ideas toward the material of the workshop which is about Action Research in Education. This fact is an interesting phenomenon showing that Indonesians do not always use turn -taking signals when they have the turn of speaking. This is done, probably, because they do not want to initiate or respond because they might assume that one of them does not give a clue for the other to response. In the conversation between two Indonesians above, for instance, $\mathbf{T}$ does not initiate the turn because $\mathbf{H}$ does not clearly ask $\mathbf{T}$ question about his idea toward the action research in education as the topic of the workshop they both attend. What $\mathbf{T}$ does above shows that using markers in turn turn-taking is not that necessarily. Two speakers might think that each of them knows when to take a turn. To the researcher observation, the Indonesians talking in English tend to take the turn of speaking when one of them is silent or making a pause. The other reason might be related to the absence of clarification or confirmation words or expressions from one of the participants. In the case between $\mathbf{T}$ and $\mathbf{H}$ above, no turn marker used by $\mathbf{T}$ when he responses what $\mathbf{H}$ says about action research in Education.

Comparing the two groups, Indonesians and Americans above, it is clear that most of them use the same markers of tum in turn-taking. However, in terms of frequency, the Americans seem to use those markers in almost every part of their conversation. Meanwhile, Indonesians are rarely used those markers in their turntaking due to the belief that, for example, making a pause is better when one is talking and taking a turn is not always necessarily using markers. They might believe that certain "gesture" could be made as a marker of a turn. All in all, what happens between the two groups as illustrated above is also supported by Tibbetts (2008) who says that showing a turn like turn- taking in a conversation varies from culture to culture. 


\section{E. Conclusion}

This writing has shown that both native (Americans) and non-native (Indonesians) speakers of English have some similarities in terms of markers used to sign a turn in their conversation. The two groups use words like yeah, but, okay, well, and so on, to indicate the turn. This shows that in terms of turn taking markers, there is no significant difference between the two groups. This fact might also be influenced by the globalization issue in which English has been widely used by many people all over the globe. This, therefore, gives more opportunities to many people in EFL areas including Indonesia to have more exposure in English.

However, what makes it different is more on the pause made during the progress of the talk and reasons of using them. Indonesians who are involved in this study, tend to make a longer pause when one of them is talking. This could be done as a way to appreciate the speaker having the talking tum due to the cultural norm they believe. In addition, this use of words signaling a turn is made as a strategy to plan what to say next, to make a new start and to reformulate what they have just said. Meanwhile, the Americans show a little bit different thing in this matter. They tend to keep signaling a turn by saying yeah, yes, so, well in every sequence of their conversation. This could be done as a form of appreciation toward the one who is talking.

\section{F. Pedagogical Implication}

The study on turn signals like turn taking markers could be one alternative way to help students of EFL understand more the nature of English as a foreign language especially its use in a casual form of conversation. Exploring these markers used by both native and non-native speakers of English, in terms of function, will give students a feel and an idea of the way these turn markers are constructed and used across different context of situations in which culture is significantly influencing them.-This way, students are more apt to build a practical repertoire of workable language resources for making a better conversation in English.

Other implication is their cross-cultural awareness. Students can leam how Americans as exemplified by the two people involved in this study, for example, use English particularly in using turn-taking markers, in their casual or formal 
conversation activities. They can also learn the social rules which somehow control the use of the language itself by observing how they select words or expressions to indicate a turn in the conversation being done. Bearing this fact, it is therefore suggested that students of EFL expand their knowledge not only on the grammar of English but also the social rules (culture) of using the language. Observing real use of English like in natural contexts of situations could be a place for their improvement.

\section{REFERENCES}

Anonym. 1996. Exploring Language: Turn Taking. (http://english. unitechnology.ac.nz/resources/resources/explang/turn taking.html. retrieved on April 14, 2010)

Aijmer, K (2005). Pragmatic Markers in Spoken Interlanguage. Amsterdam \& Philadelphia: John Benjamins.

Cook, G. 1989. Discourse. Oxford: Oxford University Press.

Levinson, Stephen C. 1983.Pragmatics. Cambridge, England: Cambridge University.

Loveday, L. 1982. The Sociolinguistics of Learning and Using a Non-native language. Oxford: Pergamon Press.

Miles, M.B., and Huberman, A.M. 1984. Qualitative Data Analysis: A source of BookofNew Methods. California: SAGE

Forrester, M.A. 2002. How to Do Conversation Analysis: a Brief Guide. A paper. Department of Psychology, university of Kent.

Rahman, Q.A. 2006. The Indonesian Speakers' Turn Signals in English Conversation: A Pragmatic Perspective. TEFLIN Journal, Volume 17 Number 2 August 2006.

Orestrom, B. 1983. Turn-taking in English conversation. Lund: Liber Forslag.

Stenstrom, A-B. 1994. An Introduction to Spoken Interaction. Longman, London and New York. 
Tibbetts, D, 2007. Conversation, Turn-taking and Culture. http://insights-into tefl.blogspot.com $/ 2007 / 02 /$ on-conversation-turn-taking-andculture.html. Retrieved on April 7,2010.

Yule, George. 1994. The Study of Language. Cambridge University Press.

Huang, Yan. 2007. Pragmatics. Oxford University Press.

Thomas, Jenny. 1995. Meaning in Interaction: An Introduction to Pragmatics. Longman Group Limited. 